\title{
ON THE CONNECTIVITY OF EFFICIENT POINT SETS
}

Abstract. The connectivity of the efficient point set and of some proper efficient point sets in locally convex spaces is investigated.

1. Introduction. An important problem in vector optimization is the connectivity of efficient point sets. Several authors have discussed this topic in finite-dimensional spaces and normed spaces (cf. [3], [6], [7], [10], [12], [15]-[16], [18], [19], [21] and [23]). Recently, Gong [8] and Song [22] studied the connectivity of the efficient point set and some proper efficient point sets with respect to the weak topology of a normed space. As an application, the connectivity of the efficient solution set and of some proper efficient solution sets for a vector optimization problem with a set-valued mapping which has weakly compact images was also discussed.

In this note, we first study the connectivity of the efficient point set and of some proper efficient point sets of a weakly compact, cone-convex subset of a locally convex space. As an application, we study the connectivity of efficient solution sets for set-valued vector optimization. Our results unify the corresponding results of [21] and [22].

2. Preliminaries. Let $Y$ be a locally convex space with topological dual space $Y^{*}$. Let $S \subset Y$ be a convex cone (i.e., $S+S \subset S$, and $\lambda S \subset S$ for every $\lambda \geq 0$ ). Denote by $S^{+}$the dual cone of $S$ :

$$
S^{+}=\left\{h \in Y^{*} \mid h(y) \geq 0 \text { for all } y \in S\right\} .
$$

Denote by $S^{+i}$ the set of all strictly positive linear functionals in $S^{+}$, i.e.,

$$
S^{+i}=\left\{h \in Y^{*} \mid h(y)>0 \text { for all } y \in S \backslash\{0\}\right\} .
$$

1991 Mathematics Subject Classification: 90C26, 90C29.

Key words and phrases: vector optimization, efficient point sets, connectivity, density. 
Let $C$ be a subset of $Y$. By $\bar{C}$ (resp. $\bar{C}^{w}$ ) and $\operatorname{int} C$ we denote the closure (resp. the closure with respect to the weak topology) and interior of $C$, respectively.

A nonempty convex subset $B$ of the convex cone $S$ is said to be a base for $S$ if $\bar{B}$ does not contain the origin and $S=\operatorname{cone}(B)=\{\lambda b \mid b \in B, \lambda \geq 0\}$.

It is clear that if $S^{+i}$ is nonempty, then $S$ is pointed, i.e., $S \cap(-S)=\{0\}$. Moreover, if $S$ is a nonempty convex cone in $Y$, then $S^{+i} \neq \emptyset$ if and only if $S$ has a base (see [13]).

Let $C$ be a subset of $Y$ and $S \subset Y$ be a closed convex pointed cone. A point $y_{0} \in C$ is said to be a positive proper efficient point of $C, y_{0} \in$ $\operatorname{Pos}(C, S)$, if there exists an $h \in S^{+i}$ such that

$$
h\left(y_{0}\right) \leq h(y) \quad \text { for all } y \in C .
$$

A point $y_{0} \in C$ is said to be:

- an efficient point of $C$ with respect to $S, y_{0} \in \mathrm{E}(C, S)$, if

$$
\left(C-y_{0}\right) \cap(-S)=\{0\} ;
$$

- a Borwein proper efficient point of $C$ with respect to $S$ (cf. [4]), $y_{0} \in \operatorname{Bo}(C, S)$, if

$$
\overline{\text { cone }}\left(C-y_{0}\right) \cap(-S)=\{0\},
$$

where $\overline{\operatorname{cone}}\left(C-y_{0}\right)$ denotes the closure of the set cone $\left(C-y_{0}\right)$;

- a Benson proper efficient point of $C$ with respect to $S$ (cf. [2]), $Y_{0} \in$ $\operatorname{Be}(C, S)$, if

$$
\overline{\mathrm{cone}}\left(C+S-y_{0}\right) \cap(-S)=\{0\} .
$$

It is easy to show that $\operatorname{Pos}(C, S) \subset \operatorname{Bo}(C+S, S)=\operatorname{Be}(C, S) \subset \operatorname{Bo}(C, S)$ $\subset \mathrm{E}(C, S)$ (cf. [9]).

Let $Y$ be a normed space. A point $y_{0} \in C$ is said to be a super efficient point of $C$ with respect to $S$ (cf. [5]) if there exists a number $M>0$ such that

$$
\overline{\operatorname{cone}}\left(C-y_{0}\right) \cap(B-S) \subset M B,
$$

where $B$ is the closed unit ball of $Y$. By $\mathrm{SE}(C, S)$ we denote the set of all super efficient points of $C$ with respect to the cone $S$.

A subset $C$ of $Y$ is said to be $S$-convex if $C+S$ is convex (cf. Yu [24]).

Let $A$ be a subset of a topological vector space $X$, and let $F: A \rightarrow Y$ be a set-valued mapping.

We say that $F$ is upper semicontinuous at $x_{0} \in A$ (see [1]) if for every open set $U$ such that $F\left(x_{0}\right) \subset U$, there exists a neighborhood $N\left(x_{0}\right)$ of $x_{0}$ such that $F(x) \subset U$ for every $x \in N\left(x_{0}\right) . \quad F$ is said to be upper semicontinuous on $A$ if $F$ is upper semicontinuous at every point $x \in A$. 
3. Connectivity of efficient point sets. In this section we shall discuss the connectivity of efficient point sets and some proper efficient point sets of a weakly compact and cone-convex subset in a locally convex space.

The following lemma will be used.

Lemma 1. Let $Y$ be a locally convex space and let $C$ be a weakly bounded subset of $Y$. Then the function $\phi:\left(C,\left.\sigma\left(Y, Y^{*}\right)\right|_{C}\right) \times\left(Y^{*}, \beta\left(Y^{*}, Y\right)\right) \rightarrow \mathbb{R}$ defined by

$$
\phi(y, h)=h(y)
$$

where $\sigma\left(Y, Y^{*}\right)$ is the weak topology on $Y$ and $\beta\left(Y^{*}, Y\right)$ is the strong topology on $Y^{*}$ (see [20]), is continuous.

Proof. Let $\left\{\left(y_{\alpha}, h_{\alpha}\right)\right\}$ be a net converging to $(y, h)$ in $C \times Y^{*}$. Then $\left\{y_{\alpha}\right\}$ tends weakly to $y$ in $C$ and $\left\{h_{\alpha}\right\}$ tends to $h$ in $\left(Y^{*}, \beta\left(Y^{*}, Y\right)\right)$. Consider the equality

$$
h_{\alpha}\left(y_{\alpha}\right)-h(y)=\left(h_{\alpha}-h\right)\left(y_{\alpha}\right)+h\left(y_{\alpha}-y\right) .
$$

Since $C$ is weakly bounded and $\left\{y_{\alpha}\right\} \in C$, it is clear that $h_{\alpha}\left(y_{\alpha}\right) \rightarrow h(y)$. Hence $\phi$ is continuous.

We recall that $y_{0} \in C$ is a point of continuity (PC point) for $C$ (see [14]) if for any 0-neighborhood $V$,

$$
y_{0} \notin \overline{\left[C \backslash\left(y_{0}+V\right)\right]}{ }^{w} .
$$

Equivalently, $y_{0} \in C$ is a PC point for $C$ if a net in $C$ converges (strongly) to $y_{0}$ whenever it converges weakly to $y_{0}$. We denote by $\mathrm{PC}(C)$ the set of all PC points of $C$.

If $C$ is a compact subset of $Y$, then $C=\mathrm{PC}(C)$.

THEOREM 3.1. Let $Y$ be a locally convex space and let $S$ be a closed convex cone in $Y$ such that the set $S^{+i}$ is nonempty. Let $C$ be a weakly compact, $S$-convex subset of $Y$ and let $\mathrm{E}(C, S) \subset \mathrm{PC}(C)$. Then $\operatorname{Pos}(C, S), \operatorname{Be}(C, S)$, $\mathrm{Bo}(C, S)$, and $\mathrm{E}(C, S)$ are connected.

Proof. The first part of the proof follows the lines of the proof of Theorem 1 of [21] or Theorem 3.1 of [22]. We present it here for completeness.

Define a set-valued mapping $G: Y^{*} \rightarrow Y$ by

$$
G(h)=\{y \in C \mid h(y)=\min \{h(z) \mid z \in C\}\} .
$$

We first show that $G(h)$ is convex for all $h \in S^{+i}$. Indeed, if $y_{1}, y_{2} \in G(h)$ then $y_{1}, y_{2} \in C$, and $r=h\left(y_{1}\right)=h\left(y_{2}\right)=\min \{h(y) \mid y \in C\}$. Since $h$ is a linear functional, we have $h\left(\lambda y_{1}+(1-\lambda) y_{2}\right)=\lambda h\left(y_{1}\right)+(1-\lambda) h\left(y_{2}\right)=r$ for all $\lambda \in[0,1]$. If $\lambda y_{1}+(1-\lambda) y_{2} \in C$, then $\lambda y_{1}+(1-\lambda) y_{2} \in G(h)$, i.e. $G(h)$ is convex. Assume that $\lambda y_{1}+(1-\lambda) y_{2} \notin C$ for some $\lambda \in[0,1]$. The convexity of $C+S$ implies that $\lambda y_{1}+(1-\lambda) y_{2} \in C+S$. Then there exists $y_{0} \in C$ satisfying $y_{0} \neq \lambda y_{1}+(1-\lambda) y_{2}$ such that $\lambda y_{1}+(1-\lambda) y_{2} \in y_{0}+S$. Since 
$h \in S^{+i}$, this implies that $r=h\left(\lambda y_{1}+(1-\lambda) y_{2}\right)>h\left(y_{0}\right)$, a contradiction. Thus $\lambda y_{1}+(1-\lambda) y_{2} \in C$ for all $\lambda \in[0,1]$.

Next we show that $G(\cdot)$ is an upper semicontinuous set-valued map from $Y^{*}$ into $Y$, where $Y^{*}$ is supplied with the strong topology $\beta\left(Y^{*}, Y\right)$.

Assume that $G(\cdot)$ is not upper semicontinuous at some $h_{0} \in Y^{*}$. Then there exist an open set $V$ in $Y$ such that $G\left(h_{0}\right) \subset V$ and a net $\left\{h_{\alpha}\right\}_{\alpha \in I}$ in $\left(Y^{*}, \beta\left(Y^{*}, Y\right)\right)$ converging to $h_{0} \in Y^{*}$ such that

$$
G\left(h_{\alpha}\right) \not \subset V \quad \text { for all } \alpha \in I .
$$

Hence, there exist some $y_{\alpha} \in G\left(h_{\alpha}\right) \backslash V$ for $\alpha \in I$, i.e., $y_{\alpha} \in C \backslash V$ and

$$
h_{\alpha}\left(y_{\alpha}\right) \leq h_{\alpha}(y) \quad \text { for all } y \in C .
$$

Since $C$ is weakly compact, without loss of generality, we may assume that $\left\{y_{\alpha}\right\}$ converges weakly to a point $y_{0} \in C$. Let $\phi:\left(C,\left.\sigma\left(Y, Y^{*}\right)\right|_{C}\right) \times$ $\left(Y^{*}, \beta\left(Y^{*}, Y\right)\right) \rightarrow \mathbb{R}$ be the function defined by

$$
\phi(y, h)=h(y) .
$$

Note that $C$ is weakly bounded. By Lemma $1, \phi$ is continuous. Hence $h_{\alpha}\left(y_{\alpha}\right)$ $\rightarrow h_{0}\left(y_{0}\right)$ and $h_{\alpha}(y) \rightarrow h_{0}(y)$ for all $y \in C$. Therefore,

$$
h_{0}\left(y_{0}\right) \leq h_{0}(y) \quad \text { for all } y \in C \text {. }
$$

This means that $y_{0} \in G\left(h_{0}\right) \subset V$ and hence $y_{0} \in \mathrm{E}(C, S)$. Since $y_{0} \in$ $\mathrm{PC}(C)$, we infer that $\left\{y_{\alpha}\right\}$ converges to $y_{0}$, a contradiction.

It is obvious that $S^{+i}$ is a connected subset of $\left(Y^{*}, \beta\left(Y^{*}, Y\right)\right)$. By Theorem 3.1 of [11], we know that $\operatorname{Pos}(C, S)=\bigcup_{h \in S^{+i}} G(h)$ is a connected set. Moreover, $\operatorname{Pos}(C, S) \subset \operatorname{Be}(C, S) \subset \operatorname{Bo}(C, S) \subset \mathrm{E}(C, S)$. By Theorems 4.4 and 3.2 of [17], we have $\mathrm{E}(C, S) \subset \overline{\operatorname{Pos}(C, S)}$. Therefore, the sets $\operatorname{Be}(C, S)$, $\mathrm{Bo}(C, S)$ and $\mathrm{E}(C, S)$ are connected.

REMARK 1. If $C$ is compact, then it is also weakly compact and $\mathrm{E}(C, S) \subset$ $\mathrm{PC}(C)$, but not conversely. For instance, the unit ball $B_{Y}$ in an infinitedimensional and uniformly convex Banach space $Y$ is weakly compact and each point of its sphere is a PC point, but the ball is not compact. Theorem 3.1 unifies and improves Theorem 1 of [21] on the connectivity of the efficient point set with respect to the norm topology of a normed space and Theorems 3.1 and 3.2 of [22] for the weak topology of a normed space.

REMARK 2. When $Y$ is a normed space, if $S$ has a closed bounded base, then the norm-interior int $S^{+}$is nonempty and it is also connected with respect to the norm topology. By the proof of Theorem 2.2 of [8], we have $\operatorname{SE}(C, S)=\bigcup_{h \in \operatorname{int} S^{+}} G(h)$. From the proof of Theorem 3.1, it is clear that $\mathrm{SE}(C, S)$ is also a connected set. In this case, int $S^{+} \subset S^{+i}$. Equality holds if, in addition, $Y$ is a reflexive Banach space, and hence $\operatorname{SE}(C, S)=\operatorname{Pos}(C, S)$. 
In a nonreflexive space, equality above does not hold in general. Therefore, in the final remark of [22], one has to add the assumption that $Y$ is reflexive.

Let $A$ be a subset of a topological vector space $X$, and let $F: A \rightarrow Y$ be a set-valued mapping. We consider the following vector optimization problem:

$$
\min F(x), \quad \text { subject to } x \in A \text {. }
$$

A point $x_{0} \in A$ is said to be an efficient (resp. Borwein proper efficient, Benson proper efficient, positive proper efficient) solution of the problem $(\mathrm{P})$ if there exists $y_{0} \in F\left(x_{0}\right)$ such that $y_{0}$ is in $\mathrm{E}(F(A), S)$ (resp. $\operatorname{Bo}(F(A), S)$, $\operatorname{Be}(F(A), S), \operatorname{Pos}(F(A), S))$.

Let us denote by $\mathrm{E}(A, S, F), \operatorname{Bo}(A, S, F), \operatorname{Be}(A, S, F)$, and $\operatorname{Pos}(A, S, F)$ the sets of all efficient, Borwein proper efficient, Benson proper efficient, and positive proper efficient solutions of the problem $(\mathrm{P})$, respectively.

When $Y$ is a normed space, the set of all super efficient solutions of $(\mathrm{P})$ will be denoted by $\mathrm{SE}(\mathrm{A}, \mathrm{F}, \mathrm{S})$, i.e.

$$
\mathrm{SE}(A, F, S)=\{x \in A \mid \exists y \in F(x) \text { such that } y \in \mathrm{SE}(F(A), S)\} .
$$

Let $A$ be a convex subset of $X$. A set-valued mapping $F: A \rightarrow Y$ is said to be $S$-convex if for all $x_{1}, x_{2} \in A$ and $t \in[0,1]$ we have

$$
t F\left(x_{1}\right)+(1-t) F\left(x_{2}\right) \subset F\left(t x_{1}+(1-t) x_{2}\right)+S .
$$

$F$ is said to be $S$-quasiconvex if $x_{1}, x_{2} \in A, y \in Y$,

$$
F\left(x_{1}\right) \cap(y-S) \neq \emptyset \quad \text { and } \quad F\left(x_{2}\right) \cap(y-S) \neq \emptyset
$$

imply

$$
F\left(t x_{1}+(1-t) x_{2}\right) \cap(y-S) \neq \emptyset \quad \text { for all } t \in[0,1] .
$$

It is easy to show that $F$ is $S$-quasiconvex if and only if

$$
F^{-1}(y-S)=\{x \in A \mid F(x) \cap(y-S) \neq \emptyset\}
$$

is convex for all $y \in Y$.

Clearly, if $F(\cdot)$ is $S$-convex, then $F$ is $S$-quasiconvex and $F(A)$ is $S$ convex. The converse is not true as was shown by an example of [21].

THEOREM 3.2. Let A be a compact subset of a Hausdorff topological vector space $X$, let $Y$ be a locally convex space and let $S$ be a closed convex cone in $Y$ such that the set $S^{+i}$ is nonempty. If $F: A \rightarrow Y$ is an upper semicontinuous set-valued mapping with compact images, $F(\cdot)$ is $S$-quasiconvex and $F(A)$ is $S$-convex, then $\operatorname{Pos}(A, S, F), \operatorname{Bo}(A, S, F), \operatorname{Be}(A, S, F)$, and $\mathrm{E}(A, S, F)$ are connected.

Proof. The proof is exactly the same as those in [21] or [22], so we omit it.

REMARK 3. Under the assumptions of Theorem 3.2, if, in addition, $Y$ is a normed space and $S$ has a closed bounded base (cf. Remark 2), by using 
a similar argument, we can prove that $\mathrm{SE}(A, F, S)=F^{-1}(E(F(A), S))$ is connected. This result generalizes Theorem 2.2 of [8] where the set-valued mapping $F$ was assumed to be $S$-convex.

Acknowledgements. The author would like to thank Professor S. Rolewicz for helpful discussions.

\section{References}

[1] J. P. Aubin and I. Ekeland, Applied Nonlinear Analysis, Wiley-Interscience, New York, 1984.

[2] H. P. Benson, An improved definition of proper efficiency for vector minimization with respect to cones, J. Math. Anal. Appl. 71 (1979), 232-241.

[3] G. R. Bitran and T. L. Magnanti, The structure of admissible points with respect to cone dominance, J. Optim. Theory Appl. 29 (1979), 573-614.

[4] J. M. Borwein, The geometry of Pareto efficiency over cones Math. Oper. Statist. Ser. Optim. 11 (1980), 235-248.

[5] J. M. Borwein and D. M. Zhuang, Super efficiency in vector optimization, Trans. Amer. Math. Soc. 338 (1993), 105-122.

[6] X. H. Gong, Connectedness of efficient solution sets for set-valued maps in normed spaces, J. Optim. Theory Appl. 83 (1994), 83-96.

[7] - Connectedness of the efficient solution set of a convex vector optimization in normed spaces, Nonlinear Anal. 23 (1994), 1105-1114.

[8] - Connectedness of super efficient solution sets for set-valued maps in Banach spaces, Math. Methods Oper. Res. 44 (1996), 135-145.

[9] A. Guerraggio, E. Molho and A. Zafferoni, On the notion of proper efficiency in vector optimization, J. Optim. Theory Appl. 82 (1994), 1-21.

[10] R. Hartley, On cone efficiency, cone convexity and cone compactness, SIAM J. Appl. Math. 34 (1978), 211-222.

[11] J.-B. Hiriart-Urruty, Images of connected sets by semicontinuous multifunctions, J. Math. Anal. Appl. 111 (1985), 407-422.

[12] Y. D. Hu and E. J. Sun, Connectedness of the efficient point set in strictly quasiconcave vector maximization, J. Optim. Theory Appl. 78 (1993), 613-622.

[13] J. Jahn, Mathematical Vector Optimization in Partially Ordered Linear Spaces, Lang, Frankfurt, 1986.

[14] B. L. Lin, P. K. Lin and S. L. Troyanski, Characterizations of denting points, Proc. Amer. Math. Soc. 102 (1988), 526-528.

[15] D. T. Luc, Theory of Vector Optimization, Springer, 1989.

[16] _, Contractibility of efficient points sets in normed spaces, Nonlinear Anal. 15 (1990), 527-535.

[17] E. K. Makarov and N. N. Rachkovski, Density theorems for generalized Henig proper efficiency, J. Optim. Theory Appl. 91 (1996), 419-437.

[18] P. H. Naccache, Connectedness of the set of nondominated outcomes in multicriteria optimization, ibid. 25 (1978), 459-467.

[19] J. W. Nieuwenhuis, Some results about nondominated solutions, ibid. 36 (1982), 289-310

[20] H. H. Schaefer, Topological Vector Spaces, Springer, New York, 1971. 
[21] W. Song, A note on connectivity of efficient point sets, Arch. Math. (Basel) 65 (1995), 540-545.

[22] -, Connectivity of efficient solution sets in vector optimization of set-valued mappings, Optimization 39 (1997), 1-11.

[23] A. R. Warburton, Quasiconcave vector maximization: connectedness of the sets of Pareto-optimal and weak Pareto-optimal alternatives, J. Optim. Theory Appl. 40 (1983), 537-557.

[24] P. L. Yu, Cone convexity, cone extreme points and nondominated solutions in decision problems with multiobjectives, ibid. 14 (1974), 319-377.

Wen Song

Department of Mathematics

Harbin Normal University

Harbin 150080, China

and

Institute of Mathematics

Polish Academy of Sciences

00-950 Warszawa, Poland

Received on 16.6.1997;

revised version on 20.10.1997 